\title{
openheart Resilience as a correlate of acute stress disorder symptoms in patients with acute myocardial infarction
}

\author{
Rebecca E Meister, ${ }^{1,2,3}$ Tania Weber, ${ }^{1}$ Mary Princip, ${ }^{1,2,3}$ Ulrich Schnyder, ${ }^{4}$ \\ Jürgen Barth, ${ }^{5}$ Hansjörg Znoj, ${ }^{3}$ Jean-Paul Schmid, ${ }^{6}$ Roland von Känel ${ }^{1,2,7}$
}

To cite: Meister RE, Weber T, Princip M, et al. Resilience as a correlate of acute stress disorder symptoms in patients with acute myocardial infarction. Open Heart 2015;2:e000261. doi:10.1136/openhrt-2015000261

Received 2 March 2015 Revised 3 September 2015 Accepted 9 September 2015

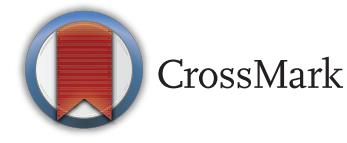

For numbered affiliations see end of article.

Correspondence to Rebecca E Meister; rebecca. meister@insel.ch

\section{ABSTRACT}

Objectives: Myocardial infarction (MI) may be experienced as a traumatic event causing acute stress disorder (ASD). This mental disorder has an impact on the daily life of patients and is associated with the development of post-traumatic stress disorder. Trait resilience has been shown to be a protective factor for post-traumatic stress disorder, but its association with ASD in patients with $\mathrm{MI}$ is elusive and was examined in this study.

Methods: We investigated 71 consecutive patients with acute MI within $48 \mathrm{~h}$ of having stable haemodynamic conditions established and for 3 months thereafter. All patients completed the Acute Stress Disorder Scale and the Resilience Scale to selfrate the severity of ASD symptoms and trait resilience, respectively.

Results: Hierarchical regression analysis showed that greater resilience was associated with lower symptoms of ASD independent of covariates $(b=-0.22, p<0.05)$.

Post hoc analysis revealed resilience level to be inversely associated with the ASD symptom clusters of re-experiencing $(b=-0.05, p<0.05)$ and arousal $(b=-0.09, p<0.05)$, but not with dissociation and avoidance.

Conclusions: The findings suggest that patients with acute MI with higher trait resilience experience relatively fewer symptoms of ASD during $\mathrm{MI}$. Resilience was particularly associated with reexperiencing and arousal symptoms. Our findings contribute to a better understanding of resilience as a potentially important correlate of ASD in the context of traumatic situations such as acute MI. These results emphasise the importance of identifying patients with low resilience in medical settings and to offer them adequate support.

\section{INTRODUCTION}

Myocardial infarction (MI) is a lifethreatening incident that may be experienced by patients as traumatic. Studies have shown that in the aftermath of an acute coronary syndrome (ACS), $18 \%$ and $16 \%$ of patients develop clinically relevant symptoms of acute stress disorder (ASD) and

\section{KEY QUESTIONS}

What is already known about this subject?

- Myocardial infarction is a life-threatening incident that may lead to acute and post-traumatic stress disorder. These disorders are associated with impairment in quality of life, social functioning, as well as cardiovascular and overall health. Several studies have shown a buffering effect of trait resilience on the development of a post-traumatic stress disorder.

What does this study add?

- On the whole, comprehensive research on protective factors for acute stress disorder is still lacking, especially in the realm of traumatic diseases. As per our knowledge, our findings are the first to show an association between trait resilience and acute stress disorder in patients with acute myocardial infarction. Our findings might contribute to a better understanding of the development and the prevention of traumatic stress after a life-threatening disease.

How might this impact on clinical practice?

- A clinical implication of our results is the importance of identifying patients especially vulnerable after a traumatic experience, such as myocardial infarction, to offer them support and psychological counselling. Appropriate, resource-oriented psychological interventions for patients low in resilience might help to prevent acute and post-traumatic stress disorder in the mid-term.

post-traumatic stress disorder (PTSD), respectively. ${ }^{1}{ }^{2}$ ASD may occur after a lifethreatening event in patients who reacted with intense fear or helplessness to it. ${ }^{3}$ ASD is characterised by symptoms assigned to the following clusters: dissociation, re-experiencing, avoidance, and increased arousal. These symptoms occur within 4 weeks and cause problems in important areas of daily life. ASD is a risk factor for the development of PTSD, which is associated with impairment in quality of life, social 
functioning, as well as cardiovascular and overall health. $^{4-6}$

Factors which may contribute to ASD include subjective distress during the traumatic situation, depression, and female sex. ${ }^{7-9}$ Severity of ACS does not seem to be a reliable predictor for the development of ASD. ${ }^{10}$ Less is known about the so-called protective factors which may mitigate the development of ASD symptoms after a potentially traumatic event. ${ }^{11}$

Resilience is one factor that is suggested to play a role in maintaining mental health in the face of adverse events, including trauma. ${ }^{12}$ Resilience is a complex construct that has been explored from different point of views. ${ }^{12} 13$ While some researchers are more interested in a resilient outcome, that is, the absence of mental disturbance after a traumatic event, others focus more on a resilient process which describes a fast adaption to a stressful event. ${ }^{12-15}$ A third perspective on resilience concerns the personality characteristics of a person who reacts well to adversities. ${ }^{13} 16$ In this paper, we are interested in the latter, namely, whether patients with a resilient personality pattern are more likely to stay psychologically well after an MI. However, the definition of this trait of resilience varies in the literature. Some authors approached resilience by related terms like optimism or self-efficacy, whereas others defined their own concept. ${ }^{13} 16$ In the present study, we rely on the comprehensive definition of Wagnild and Young. ${ }^{16}$ These authors integrated qualitative data with previous considerations on resilience from the literature; specifically, they developed a description of resilience as a stable construct over time based on the two aspects of personal competence (eg, self-reliance, independence, or resourcefulness) and acceptance of self and life (eg, flexibility and balance). ${ }^{16} 17$

Several studies have shown a predictive value of trait resilience for the development of PTSD. For instance, in a sample of patients referred to an emergency department, resilience was found to be predictive of PTSD symptom severity between 5 and 12 weeks posttrauma. ${ }^{18}$ To our knowledge, only one study has examined an association between resilience and ASD with, however, puzzling findings: In patients with mild traumatic brain injury, while no relation between resilience and ASD was found at baseline, higher resilience was associated with more severe ASD symptoms 1 week and 1 month after the traumatic incident. ${ }^{19}$ On the whole, comprehensive research on protective factors for ASD is still lacking, especially in the realm of traumatic diseases.

Therefore, the aim of our study was to find if resilience has a buffering effect on the development of ASD symptoms in patients after the traumatic experience of an acute MI. We hypothesised that resilience would inversely contribute to ASD symptoms, after controlling for demographic and medical variables. Furthermore, we wanted to explore if resilience is linked to specific ASD symptom clusters.
METHODS

\section{Study participants and design}

This study is part of the ongoing project Myocardial Infarction-Stress Prevention Intervention (MI-SPRINT).$^{20}$ The study has been approved by the ethics committee of the State of Bern, Switzerland. Data for the following analysis were consecutively collected between January 2013 and September 2014. Patients who were referred to the Coronary Care Unit (CCU) of the Bern University Hospital, Switzerland, with a ST-elevation (STEMI) or non-ST-elevation index myocardial infarction (Non-STEMI) were eligible to participate in the study. Within $48 \mathrm{~h}$ after stable haemodynamic conditions were established, patients were invited to participate in the study. All participants gave written informed consent to the study protocol. Inclusion criteria were age over 18 years, stable circulatory condition, and a certain amount of distress during MI (ie, experienced their MI as a traumatic situation; for further information see psychometric assessment). Specific exclusion criteria were emergency coronary artery bypass graft surgery, any severe comorbid disease likely to cause death within 1 year, current severe depression based on clinical judgment, lack of complete orientation or cognitive impairment, insufficient knowledge of German, suicidal ideations in the last 2 weeks, and participation in another randomised controlled trial run by the Cardiology Department.

Patients completed the Acute Stress Disorder Scale (ASDS) within $48 \mathrm{~h}$ of the acute event. Demographic and medical data were collected through medical charts and standard interviews. Based on the assumption that resilience is a stable construct over time, it was measured after a follow-up of 3 months to limit burden on patients in the acute inhospital setting.

Of the original 111 patients included in the study, 71 were available for the final analysis (figure 1). Numbers and reasons for drop-out were: 19 did not fill in questionnaires at admission due to their physical condition, refusal, or immediate transfer to other hospitals; medical and demographic information from 1 patient was missing; 5 died within the first 3 months after hospital admission; 7 could not be reached for the 3-month follow-up assessment; 7 refused further participation after 3 months; and 1 did not fill in the resilience questionnaire.

\section{Psychometric assessment}

\section{ASD symptoms}

ASD symptoms were measured with the validated German version of the ASDS. ${ }^{21} 22$ The ASDS is a 19-item self-report inventory which comprises the four subscales dissociation (5 items), re-experiencing (4 items), avoidance (4 items) and arousal (6 items) based on Diagnostic and Statistical Manual of Mental Disorders the (DSM-IV) criteria for ASD. ${ }^{3}$ Each item is rated on a five-point Likert scale ( $0=$ 'not at all,' $4=$ ='extremely)' with a sum score between 0 and 76 . All patients were asked to 


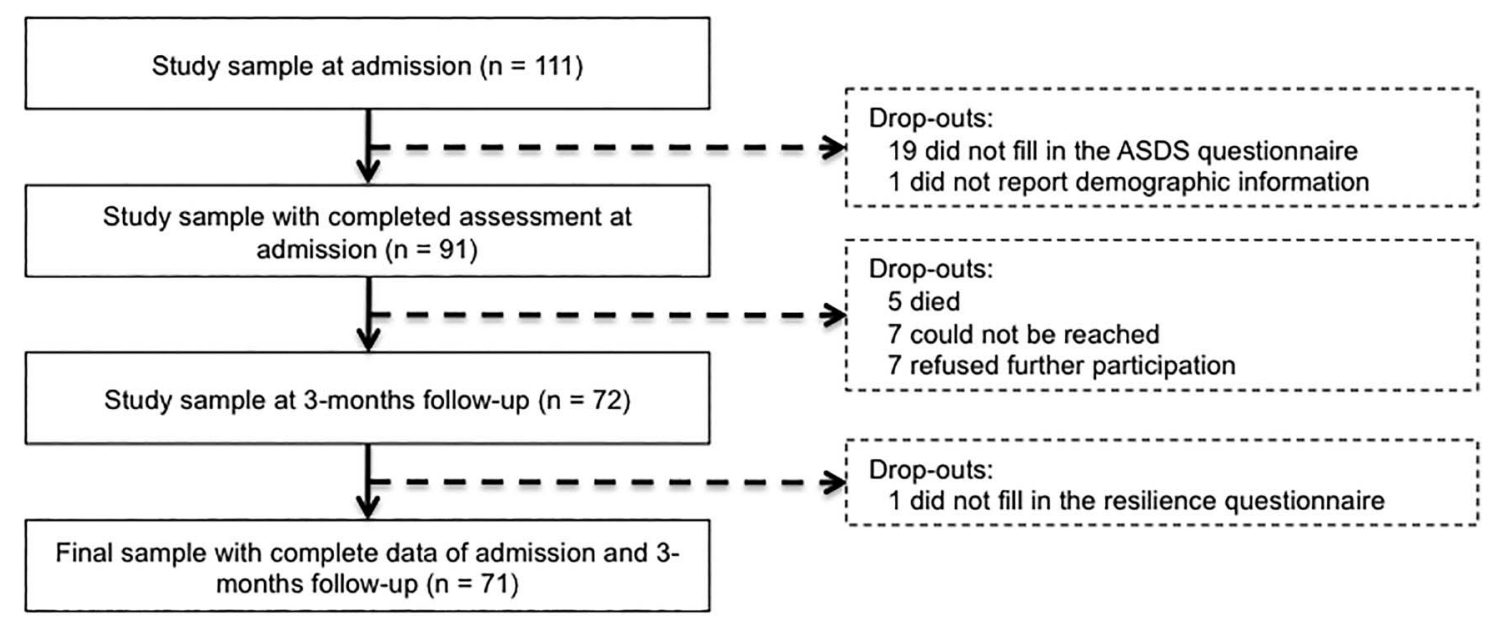

Figure 1 Participant flow with numbers and reasons for drop-out; ASDS, Acute Stress Disorder Scale.

rate the questionnaire with respect to the cardiac event. The ASDS has been validated for a cardiac sample with satisfactory to good internal consistency for the total scale and subscales (Cronbach's $\alpha$ for total scale $=0.88$, dissociation $=0.89$, re-experiencing $=0.78$, avoidance $=0.62$, arousal $=0.62) .{ }^{22}$ We found comparable reliability in our sample (Cronbach's $\alpha$ for total scale $=0.83$, dissociation $=0.65$, re-experiencing $=0.63$, avoidance $=0 . \quad 56$, arousal $=0.74$ ).

\section{Resilience}

Trait resilience was assessed with the German short version of the Resilience Scale. ${ }^{16}{ }^{17}$ This self-rating instrument consists of 11 items scored on a seven-point Likert scale (1='disagree,' $7=$ 'agree') with a sum score between 11 and 77 . Typical items are 'I usually manage one way or another' and 'I can usually look at a situation in a number of ways.' The original 25-items form showed a two-factorial structure, that is, personal competence and acceptance of self and life. Schumacher et al ${ }^{17}$ constructed an economical short form with items from both scales, which resulted in a stable one-factorial structure, with good internal consistency (Cronbach's $\alpha=0.91$ ). Similar reliability was found in our sample (Cronbach's $\alpha=0.93$ ).

\section{Covariates}

Data on age, educational level, medical history, smoking, weight and height were obtained by a standard clinical interview and completed from medical charts when missing. MI-triggered distress was assessed with three single-item questions asking about the intensity of pain, fear of dying and helplessness to be rated on a numeric scale ranging from 0 to 10 . Similar questions have been used in previous studies and these show a valid assessment of the subjective peritraumatic experience during MI. ${ }^{63}$ Only patients who scored at least 5 for chest pain plus at least 5 for fear of dying and/or helplessness were considered to have experienced the MI as a traumatic event and thus, were included in the study. For further analysis, we calculated a sum score of the three items. Data on troponin $\mathrm{T}$ peak levels, MI specification data, left ventricular ejection fraction, Killip class and the number of diseased vessels with at least $50 \%$ coronary lumen stenosis were obtained from hospital charts.

\section{Statistical analysis}

Data were analysed using PASW V.21.0 statistical software package (SPSS Inc., Chicago, Illinois, USA). Level of significance was set at $\mathrm{p}<0.05$ (two-tailed). Normal distribution was verified by the Kolmogorov-Smirnov test. The ASDS subscale 'dissociation' had to be square root transformed to approximate a normal distribution before analysis. For all psychometric scales, we replaced missing item values by the expectation maximisation algorithm if at least $70 \%$ of the items of that scale were answered. ${ }^{24}$ To illustrate participant's characteristics as per resilience, a median split on this variable was conducted. The two groups below and above the median were compared using one-way analysis of variance and Pearson $\chi^{2}$ test for continuous and categorical variables, respectively.

Hierarchical regression analysis with forced entry of covariates was applied first, to test the independent contribution of resilience to the ASDS sum score and second, to perform post hoc analyses for each of the ASDS subscale scores separately. Assumptions of linearity, homoscedasticity and exclusion of multicollinearity were assured by scatter plots and curve estimations. Exclusion of autocorrelation was done by Durbin Watson statistic. Regarding the total sample of 71 patients, no regression equation considered more than seven covariates to avoid overfitted and thus unstable models. We entered, as a first step, the a priori defined control variables age, gender and educational status. In the second step, we entered peritraumatic factors (ie, distress level and troponin $\mathrm{T}$ peak) and medical history (ie, previous MI and history of depression) in the model. In the last step, resilience was entered into the equation. We displayed unstandardised b coefficients, SEs of the mean (SEM) and changes in $\mathrm{R}^{2}$ of each step with $\mathrm{p}$ values. 


\section{RESULTS}

\section{Patient characteristics}

Table 1 shows the characteristics of all patients as per resilience category. The average age of the 71 patients included into the analysis was 58 years and the majority was male. There were no significant differences in sociodemographic and biomedical variables between the two groups. No participant fulfilled full criteria of an ASD.

\section{Regression analysis for ASDS sum score}

Table 2 shows the hierarchical linear regression model to determine independent predictors of the ASDS sum score. In step 1, neither gender nor age nor education made a significant contribution to the outcome. In step 2 , only distress level was significantly associated with the ASDS sum score such that the distressed patients had a higher level on the ASDS $(b=0.90, p<0.01)$, with the model explaining almost $20 \%$ of the variance. Resilience, which was entered in step 3, emerged as a significant and inverse predictor of ASD symptom levels $(b=-0.22, p<0.05)$ such that patients with more resilience showed lower ASDS scores. Resilience explained an additional variance of $7 \%$ of the outcome variable after controlling for all other covariates in the final model.

\section{Post hoc analysis of ASDS subscale scores}

We analysed post hoc the individual dimensions of the ASDS to identify those to be particularly predicted by resilience. Table 3 shows the fully adjusted hierarchical linear regression models for each of the four ASDS subscales. In the first regression equation, only distress emerged as an independent predictor of dissociative symptoms $(b=0.27, p<0.05)$. The second regression equation revealed distress $(\mathrm{b}=0.20, \mathrm{p}<0.05)$ and resilience $(b=-0.05, p<0.05)$ to be independently associated with re-experiencing, with resilience explaining $5 \%$ of the variance. The third regression equation revealed no significant association between resilience and avoidance symptoms. In the fourth regression equation, we found again distress level $(\mathrm{b}=0.32, \mathrm{p}<0.01)$ and resilience $(b=-0.09, p<0.05)$ to be independent predictors of arousal symptom levels, with resilience explaining $7 \%$ of the variance.

Table 1 Characteristics of all patients $(\mathrm{N}=71)$ and per high and low resilience

\begin{tabular}{|c|c|c|c|c|}
\hline Variables & All $(\mathrm{N}=71)$ & Low resilience $(n=35)$ & High resilience $(n=36)$ & p Value \\
\hline Age (years) & $57.8 \pm 9.8$ & $58.2 \pm 10.0$ & $57.3 \pm 9.7$ & 0.703 \\
\hline Male gender (\%) & 77.5 & 74.3 & 80.6 & 0.527 \\
\hline Highest level of education (\%) & & & & 0.180 \\
\hline Primary school & 8.5 & 11.4 & 5.6 & \\
\hline Vocational school & 73.2 & 80.0 & 66.7 & \\
\hline College & 4.2 & 2.9 & 5.6 & \\
\hline University & 14.1 & 5.7 & 22.2 & \\
\hline Body mass index $\left(\mathrm{kg} / \mathrm{m}^{2}\right)$ & $28.2 \pm 5.3$ & $27.4 \pm 5.6$ & $29.0 \pm 5.0$ & 0.221 \\
\hline Hypertension (\%) & 54.9 & 54.3 & 55.6 & 0.914 \\
\hline Hypercholesterolemia (\%) & 49.3 & 42.9 & 55.6 & 0.285 \\
\hline Diabetes (\%) & 11.3 & 14.3 & 8.3 & 0.428 \\
\hline Smoking (\%) & 42.3 & 37.1 & 47.2 & 0.390 \\
\hline Previous MI (\%) & 8.5 & 8.6 & 8.3 & 0.971 \\
\hline $\begin{array}{l}\text { Positive family history of } \\
\text { coronary artery disease (\%) }\end{array}$ & 35.2 & 42.9 & 27.8 & 0.184 \\
\hline History of depression (\%) & 22.5 & 28.6 & 16.7 & 0.230 \\
\hline Myocardial infarction (\%) & & & & 0.327 \\
\hline STEMI & 71.8 & 77.1 & 66.7 & \\
\hline Non-STEMI & 28.2 & 22.9 & 33.3 & \\
\hline Number of diseased vessels (\%) & & & & 0.287 \\
\hline 0 vessel & 1.4 & 0.0 & 2.8 & \\
\hline 1 vessel & 39.4 & 31.4 & 47.2 & \\
\hline 2 vessel & 33.8 & 42.9 & 25.0 & \\
\hline 3 vessel & 25.4 & 25.7 & 25.0 & \\
\hline Killip classification (\%) & & & & 0.805 \\
\hline Killip I & 87.3 & 88.6 & 86.1 & \\
\hline Killip II & 8.5 & 8.6 & 8.3 & \\
\hline Killip III & 1.4 & 0.0 & 2.8 & \\
\hline Killip IV & 2.8 & 2.9 & 2.8 & \\
\hline Troponin T peak ( $\mu \mathrm{g} / \mathrm{L})$ & $4.0 \pm 4.5$ & $5.0 \pm 5.5$ & $3.1 \pm 3.0$ & 0.078 \\
\hline LVEF (\%) & $48.5 \pm 11.9$ & $48.9 \pm 12.6$ & $48.1 \pm 11.3$ & 0.778 \\
\hline
\end{tabular}

Data are shown as mean \pm SD or $\%$.

LVEF, left ventricular ejection fraction; MI, myocardial infarction; STEMI, ST-segment elevation myocardial infarction. 
Table 2 Hierarchical regression analysis with acute stress disorder scale sum score as the outcome variable

\begin{tabular}{llrr}
\hline Variables entered & Step 1 & Step 2 & Step 3 \\
\hline Model statistics & $\Delta \mathrm{R}^{2}=0.004$ & $\Delta \mathrm{R}^{2}=0.189$ & $\Delta \mathrm{R}^{2}=0.067$ \\
& $\mathrm{p}=0.963$ & $\mathrm{p}=0.051$ & 0.012 \\
Age & $-0.05 \pm 0.12$ & $0.03 \pm 0.12$ & $0.05 \pm 0.12$ \\
Gender & $-0.47 \pm 2.90$ & $-2.40 \pm 2.91$ & $-1.86 \pm 2.82$ \\
Education level & $0.28 \pm 1.51$ & $0.42 \pm 1.41$ & $1.17 \pm 1.40$ \\
Distress & & $0.90 \pm 0.32^{* *}$ & $0.95 \pm 0.31^{* *}$ \\
Troponin T peak & & $0.03 \pm 0.28$ & $-0.06 \pm 0.27$ \\
Previous MI & & $1.38 \pm 4.26$ & $1.70 \pm 4.12$ \\
History of depression & $4.85 \pm 2.84$ & $3.76 \pm 2.78$ \\
Resilience & & $-0.22 \pm 0.09^{*}$ \\
\hline Data are shown as unstandardised b coefficients \pm SEM. Significance level for $p$ values: ${ }^{*} p<0.05,{ }^{* *} p<0.01,{ }^{* * *} p<0.001$.
\end{tabular}

\section{DISCUSSION}

We found higher levels of resilience to be related to lower scores on the ASDS, whereby resilience explained $7 \%$ of the sum score of ASD symptoms independently of demographic, peritraumatic and medical factors. This result is in line with previous findings on the role of resilience in other traumatic reactions such as the development of PTSD. ${ }^{18}$ To our knowledge, the only study that had considered ASD as an outcome in relation to resilience showed a puzzling finding, namely, higher resilience was found to be associated with higher ASD levels. ${ }^{19}$ The authors explain their unexpected result by suggesting the possibility of a missing mediator variable or occurrence of post-traumatic growth within 1 month after the trauma. That study not only differed from ours in the investigated trauma situation (ie, mild traumatic brain injury), but also in the instrument used for the measurement of trait resilience (ie, Connor-Davidson Resilience Scale). ${ }^{25}$ This divergent methodology might possibly account for the differing findings.

Our results may suggest that resilient patients will not only be at lower risk to develop post-traumatic stress, but that a stress-buffering effect sets in very early after the trauma, as is evidenced by their relatively lower levels of ASD symptoms. Future studies should investigate whether resilient persons can better cope with stressful events or experience the event per se as less stressful.

Our post hoc analysis revealed that there was a significant inverse correlation between resilience and the ASDS subscales re-experiencing and arousal, but not with the subscales dissociation and avoidance. Dissociation and avoidance may be seen as ASD symptoms that downregulate the stress system, leading to numbing and behavioural inhibition of the patient. Conversely, re-experiencing and arousal are more active symptoms of ASD, with patients being more agitated. Therefore, trait characteristics of resilience, that is, personal competence and acceptance of self and life, seem to be particularly important for buffering the psychophysiological overactivation of the organism after a traumatic event. In contrast, resilience may have less of an impact on freezing symptoms. However, these assumptions and a potential impact on cardiac health require further investigation.

We further found that demographic factors did not significantly correlate with ASD symptoms. As yet, little is known about the predictive value of demographic factors for ASD or PTSD, with some studies indicating an association with gender, age or education. ${ }^{8}{ }^{26}$ In our study, most of the subjects were older men, thereby limiting inferences from our findings to this specific population.

Among the peritraumatic factors, subjective experience of the MI, that is, distress level, was significantly associated with ASD symptoms, whereas an objective measure of MI damage (ie, troponin $\mathrm{T}$ peak level) was not. Peritraumatic distress (ie, pain, fear of dying and/ or helplessness) accounted for almost $20 \%$ of the variance in the outcome variable. This finding is consistent with previous research on ASD and PTSD in patients with acute MI. ${ }^{10} 27$

A medical history of previous MI and a depressive episode was not significantly predictive for the ASDS sum score. Studies on the influence of recurrent MI on the development of ASD are rare, with some studies suggesting that the risk of developing PTSD might be increased. ${ }^{28}$ On the other hand, patients who had previously experienced an MI might perhaps feel less overwhelmed by the medical situation as they are already 'familiar' with the procedures in the clinical setting of a CCU; however, this would need to be confirmed in studies. Depression is known to be a predictor of ASD. ${ }^{927}$ One possible reason why we could not replicate this finding might be that we excluded patients with a current severe depressive episode.

Our study has several limitations. Owing to the small sample size, we had to restrict the number of covariates to avoid overfitting in the statistical model. However, based on studies on PTSD, there might be other important covariates of ASD, such as social support, history of psychiatric disorders other than depression, previous life events, all of which might partially account for the resilience-ASD relationship. ${ }^{9} 1127$ We focused on covariates with the greatest relevance as reported in available 
Table 3 Post hoc hierarchical regression analysis with subscale scores of acute stress disorder scale as outcome variables

\begin{tabular}{|c|c|c|c|c|c|}
\hline \multirow[b]{2}{*}{ Variables entered } & \multirow[b]{2}{*}{ b } & \multirow[b]{2}{*}{ p Value } & \multicolumn{2}{|l|}{$95 \% \mathrm{Cl}$} & \multirow[b]{2}{*}{$\mathbf{R}^{2}$} \\
\hline & & & Lower bound & Upper bound & \\
\hline Dissociation as outcome variable & & & & & 0.166 \\
\hline Gender & $-0.59 \pm 0.31$ & 0.059 & -1.212 & 0.024 & \\
\hline Education level & $0.08 \pm 0.15$ & 0.593 & -0.224 & 0.389 & \\
\hline Distress & $0.07 \pm 0.03$ & $0.037^{*}$ & 0.004 & 0.139 & \\
\hline History of depression & $0.33 \pm 0.31$ & 0.279 & -0.277 & 0.943 & \\
\hline Resilience & $-0.02 \pm 0.01$ & 0.150 & -0.035 & 0.006 & \\
\hline Re-experiencing as outcome variable & & & & & 0.238 \\
\hline Age & $-0.03 \pm 0.03$ & 0.427 & -0.088 & 0.038 & \\
\hline Gender & $0.60 \pm 0.77$ & 0.438 & -0.942 & 2.150 & \\
\hline History of depression & $0.97 \pm 0.76$ & 0.207 & -0.553 & 2.499 & \\
\hline Resilience & $-0.05 \pm 0.03$ & $0.047^{*}$ & -0.103 & -0.001 & \\
\hline Avoidance as outcome variable & & & & & 0.105 \\
\hline Age & $-0.02 \pm 0.04$ & 0.647 & -0.086 & 0.054 & \\
\hline Gender & $0.41 \pm 0.85$ & 0.634 & -1.297 & 2.115 & \\
\hline Education level & $-0.09 \pm 0.42$ & 0.833 & -0.935 & 0.756 & \\
\hline Distress & $0.17 \pm 0.09$ & 0.072 & -0.015 & 0.335 & \\
\hline Troponin T peak & $0.01 \pm 0.08$ & 0.949 & -0.157 & 0.167 & \\
\hline Previous MI & $0.50 \pm 1.25$ & 0.690 & -1.995 & 2.995 & \\
\hline History of depression & $0.41 \pm 0.84$ & 0.629 & -1.275 & 2.093 & \\
\hline Resilience & $-0.03 \pm 0.03$ & 0.364 & -0.082 & 0.031 & \\
\hline Resilience & $-0.09 \pm 0.04^{*}$ & $0.018^{*}$ & -0.158 & -0.015 & \\
\hline
\end{tabular}

literature. $^{7-9}$ Another limitation is the assessment of resilience at 3 months in order to limit burden imposed by the MI-SPRINT protocol on patients during the acute phase of a severe medical condition. Even if resilience is a stable trait characteristic of a given individual, we cannot exclude the possibility that it was influenced by emotional and cognitive processes during recovery from and adaptation to the cardiac disease. The crosssectional design of our study does not allow for causal inferences or the conduction of a formal mediator analysis, so a prospective design would be needed to clarify if resilience or peritraumatic distress has any impact on ASD or vice versa. As participation in our study was voluntary, our sample might be slightly biased. Although all patients met the cut-off for clinically relevant distress, they especially rated fear of dying and helplessness on the lower end of the required score and might, hence, be comparably less distressed than patients who refused participation. Our study started before the release of DSM-5 in May 2013 and we, therefore, focused on the DSM-IV criteria of ASD. ${ }^{3}{ }^{29}$ Whether our findings hold for DSM-5 criteria would need a replication of this study.

A clinical implication of our results is the importance of identifying patients especially vulnerable after a traumatic experience, such as MI, to offer them support and psychological counselling. We could confirm findings of previous studies regarding distress during traumatic event to be associated with higher ensuing stress levels. Additionally, based on our results, it seems conceivable that patients with low traits of resilience are also more susceptible to develop acute stress after MI. Appropriate, resource-oriented psychological interventions for patients low in resilience might help prevent acute and PTSD in the mid-term. 
In sum, our study showed an inverse association of resilience with ASD symptom levels in patients with acute MI independent of demographic and medical factors, including cardiac disease severity. Relevant association with the ASDS score was also observed for perceived trauma severity, for example, pain and fear/ helplessness. Therefore, it seems appropriate to identify patients with low resilience in medical settings so that adequate support can be offered. Altogether, our study is a step forward towards a better understanding of resilience as a potentially important correlate of ASD in the context of traumatic situations such as acute MI.

\section{Author affiliations}

${ }^{1}$ Department of Neurology, Inselspital, Bern University Hospital, and University of Bern, Bern, Switzerland

${ }^{2}$ Psychosomatic Research Group, Department of Clinical Research, University of Bern, Bern, Switzerland

${ }^{3}$ Division of Clinical Psychology and Psychotherapy, Institute of Psychology, University of Bern, Bern, Switzerland

${ }^{4}$ Department of Psychiatry and Psychotherapy, University Hospital Zurich, University of Zurich, Zurich, Switzerland

${ }^{5}$ Institute for Complementary and Integrative Medicine, University Hospital Zurich, University of Zurich, Zurich, Switzerland

${ }^{6}$ Department of Cardiology, Bern University Hospital and Spital Netz Bern, Spital Tiefenau, Bern, Switzerland

${ }^{7}$ Department of Psychosomatic Medicine, Clinic Barmelweid, Barmelweid, Switzerland

Acknowledgements The authors wish to thank Annette Kocher for editorial assistance, and Miriam Koemeda, Christina Gattlen, Marco Scholz, and Laurin Imholz for their help in data collection.

Contributors RvK, JPS, US, JB, and HZ designed the study. REM, MP and RVK performed the statistical analysis. REM wrote the first draft of the manuscript. All authors critically revised and approved the final manuscript. RvK is the guarantor for the study.

Funding This research was supported by grant 140960 from the Swiss National Science Foundation to RvK (PI), JPS, US, HZ and JB.

Competing interests None declared.

Ethics approval Ethics approval was obtained from the ethics committee of the State of Bern, Switzerland.

Provenance and peer review Not commissioned; externally peer reviewed.

Data sharing statement No additional data are available.

Open Access This is an Open Access article distributed in accordance with the Creative Commons Attribution Non Commercial (CC BY-NC 4.0) license, which permits others to distribute, remix, adapt, build upon this work noncommercially, and license their derivative works on different terms, provided the original work is properly cited and the use is non-commercial. See: http:// creativecommons.org/licenses/by-nc/4.0/

\section{REFERENCES}

1. Edmondson D, Richardson S, Falzon L, et al. Posttraumatic stress disorder prevalence and risk of recurrence in acute coronary syndrome patients: a meta-analytic review. PLOS ONE 2012;7: e38915

2. Ginzburg K, Solomon Z, Koifman B, et al. Trajectories of posttraumatic stress disorder following myocardial infarction: a prospective study. J Clin Psychiatry 2003;64:1217-23.

3. American Psychiatric Association. Diagnostic and statistical manual of mental disorders. 4th edn. Washington DC: American Psychiatric Association, 1994.
4. Bryant RA. Acute stress disorder as a predictor of posttraumatic stress disorder: a systematic review. J Clin Psychiatry 2011;72:233-9.

5. Ginzburg K, Ein-Dor T. Posttraumatic stress syndromes and health-related quality of life following myocardial infarction: 8-year follow-up. Gen Hosp Psychiatry 2011;33:565-71.

6. von Känel R, Hari R, Schmid JP, et al. Distress related to myocardia infarction and cardiovascular outcome: a retrospective observational study. BMC Psychiatry 2011;11:98-105.

7. Bryant RA, Panasetis P. Panic symptoms during trauma and acute stress disorder. Behav Res Ther 2001;39:961-6.

8. McCarthy MC, Ashley DM, Lee KJ, et al. Predictors of acute and posttraumatic stress symptoms in parents following their child's cancer diagnosis. J Trauma Stress 2012;25:558-66.

9. Roberge MA, Dupuis G, Marchand A. Acute stress disorder after myocardial infarction: prevalence and associated factors. Psychosom Med 2008;70:1028-34.

10. Ginzburg K, Solomon Z, Bleich A. Repressive coping style, acute stress disorder, and posttraumatic stress disorder after myocardial infarction. Psychosom Med 2002;64:748-57.

11. Cardeña E, Carlson E. Acute stress disorder revisited. Annu Rev Clin Psychol 2011;7:245-67

12. Luthar SS, Cicchetti D, Becker B. The construct of resilience: a critical evaluation and guidelines for future work. Child Dev 2000;71:543-62.

13. Southwick SM, Bonanno GA, Masten AS, et al. Resilience definitions, theory, and challenges: interdisciplinary perspectives. Eur J Psychotraumatol 2014;5:25338.

14. Agaibi CE, Wilson JP. Trauma, PTSD, and resilience: a review of the literature. Trauma Violence Abuse 2005;6:195-216.

15. Rutter M. Resilience in the face of adversity. Protective factors and resistance to psychiatric disorder. Br J Psychiatry 1985;147:598-611.

16. Wagnild GM, Young HM. Development and psychometric evaluation of the resilience scale. J Nurs Meas 1993;1:165-78.

17. Schumacher J, Leppert K, Gunzelmann T, et al. Die Resilienzskala -Ein Fragebogen zur Erfassung der psychischen Widerstandsfähigkeit als Personmerkmal. Z Klin Psychol Psychiatr Psychother 2005;53:16-39.

18. Daniels JK, Hegadoren KM, Coupland NJ, et al. Neural correlates and predictive power of trait resilience in an acutely traumatized sample: a pilot investigation. J Clin Psychiatry 2012; 73:327-32.

19. McCauley SR, Wilde EA, Miller ER, et al. Preinjury resilience and mood as predictors of early outcome following mild traumatic brain injury. J Neurotrauma 2013;30:642-52.

20. Meister R, Princip M, Schmid JP, et al. Myocardial Infarction-Stress PRevention INTervention (MI-SPRINT) to reduce the incidence of posttraumatic stress after acute myocardial infarction through trauma-focused psychological counseling: study protocol for a randomized controlled trial. Trials 2013;14:329-39.

21. Bryant RA, Moulds ML, Guthrie RM. Acute stress disorder scale: a self-report measure of acute stress disorder. Psychol Assess 2000;12:61-8.

22. Helfricht $\mathrm{S}$, Landolt MA, Moergeli $\mathrm{H}$, et al. Psychometric evaluation and validation of the German version of the acute stress disorde scale across two distinct trauma populations. J Trauma Stress 2009;22:476-80.

23. Whitehead DL, Strike P, Perkins-Porras L, et al. Frequency of distress and fear of dying during acute coronary syndromes and consequences for adaptation. Am J Cardiol 2005;96:1512-16.

24. Do CB, Batzoglou $\mathrm{S}$. What is the expectation maximization algorithm? Nat Biotechnol 2008;26:897-9.

25. Connor KM, Davidson JR. Development of a new resilience scale: the Connor-Davidson resilience scale (CD-RISC). Depress Anxiety 2003;18:76-82.

26. Brewin CR, Andrews B, Valentine JD. Meta-analysis of risk factors for posttraumatic stress disorder in trauma-exposed adults. J Consult Clin Psychol 2000;68:748-66.

27. von Känel R, Gander ML. Post-traumatic stress disorder: emerging risk factor and mechanisms. In: Hjemdahl P, Rosengren A, Steptoe A, eds. Stress and cardiovascular disease. London, UK: Springer, 2012:235-56.

28. Kutz I, Shabtai H, Solomon Z, et al. Post-traumatic stress disorder in myocardial infarction patients: prevalence study. Isr J Psychiatry Relat Sci 1994;31:48-56.

29. American Psychiatric Association. Diagnostic and statistical manual of mental disorders. 5th edn. Washington DC: American Psychiatric Association, 2013 